\title{
WCC 2016-105: Risk Stratification of Women with Peripartum Cardiomyopathy at Initial Presentation: A Dobutamine Stress Echocardiography Study
}

R V Venkata Rao, G Ramesh, D Seshagiri Rao, O Sai Satish, LSR Krishna, M Adithya.

Objectives: We sought to determine the prognostic use of inotropic contractile reserve on risk stratification and prognostication of women with peripartum cardiomyopathy.

Background: Peripartum cardiomyopathy is a rare disorder effecting women in their prime years of life. There appears to be an initial high-risk period with $25 \%$ to $50 \%$ of women dying within the first 3 months postpartum. Early risk stratification and prognostication are, thus, crucial. However, only limited data are available.

Methods: In all, 10 women (mean age 20.7 years) with peripartum cardiomyopathy and severe left ventricular (LV) dysfunction (mean LV ejection fraction [LVEF] $27.3+6.5 \%$ ) were studied. Of these, 8 underwent dobutamine stress echocardiography at baseline and a follow-up resting echocardiogram at a mean of $3.6+0.9$ months after initial presentation. Resting and peak inotropic contractile reserve, and follow-up LVEF, were computed.

Results: The mean LVEF improved significantly from baseline $(27.3+6.5 \%)$ to maximal inotropic contractile reserve $(52.6+11.2 \%)\left(\mathrm{P}_{\ldots} .0004\right)$ and at follow-up $\left(54.2 \ldots\right.$ 14.3\%) $\left(\mathrm{P}_{-} .006\right)$. Importantly, LVEF at maximal inotropic contractile reserve and at follow-up (3.6 months) did not differ significantly. The mean LVEF at maximal inotropic contractile reserve correlated well with the follow- up (LVEF R_ 0.79). However, the baseline LVEF did not correlate with follow-up LVEF.

Conclusions: In patients presenting with peripartum cardiomyopathy, inotropic contractile reserve during dobutamine stress echocardiography accurately correlates with subsequent recovery of LV function and confers a benign prognosis.

$R$ V Venkata Rao ${ }^{l}$, G Ramesh $^{2}$, D Seshagiri Rao ${ }^{2}$, O Sai Satish $^{2}$, LSR Krishna ${ }^{2}$, M Adithya $^{2}$,

${ }^{I} D M$ Student, Department Of Cardiology, NIMS, Hyderabad, India,

${ }^{2}$ Department of Cardiology, NIMS.

Corresponding Author: RV Venkata Rao,

Email:prathyuam@gmail.com 